\title{
ON EXPLICIT RANDOM-LIKE TOURNAMENTS
}

\author{
SHOHEI SATAKE
}

\begin{abstract}
We give a new theorem describing a relation between the quasi-random property of regular tournaments and their spectra. This provides many solutions to a constructing problem mentioned by Erdös and Moon (1965) and Spencer (1985).
\end{abstract}

\section{INTRODUCTION}

A tournament is an oriented complete graph. Random tournaments $\mathcal{T}_{n}$ with $n$ vertices are obtained by choosing a direction of each edge of a complete graph with $n$ vertices with probability $1 / 2$, independently. We say that random tournaments asymptotically almost surely (a.a.s.) satisfy a property $\mathcal{P}$ if the probability of the event that tournaments satisfy $\mathcal{P}$ tends to 1 when $n$ goes to infinity. In graph theory, there have been many problems focusing on deterministic tournaments satisfying properties which random tournaments a.a.s satisfy; see e.g. [1], 4], 8], [9], [19].

In this paper, as such a property, we mainly focus on the quasi-random property proposed by Chung-Graham [8]. Our main result is to give a new theorem describing a relation between the quasi-random property and spectra of regular tournaments. This result also provides many solutions to a problem, proposed by Erdős-Moon [14] and Spencer [31] (see also [1, Section 9.1]), on explicit constructions of tournaments with a small number of consistent edges. It is well-known that Paley tournaments have the quasirandom property (e.g. [8]). Moreover, by proving that Paley tournaments have a property stronger than the quasi-random property, Alon-Spencer [1] showed that they provide solutions to the problem by Erdös, Moon and Spencer. We note that the proof in [1] contains a part (Lemma 9.1.2 in [1]) depending on the definition of Paley tournaments. Remarkably, we generalize their discussion to all regular tournaments by using a digraph-version of the expander-mixing lemma proved by $\mathrm{Vu}$ [33].

The rest of this paper is organized as follows. In Section 2, we recap the quasi-random property and introduce some related known facts. In Section 3, we introduce our main result and give its proof. In Section 4, we

2010 Mathematics Subject Classification. 05C20.

Key words and phrases. Eigenvelues, expander-mixing lemma, the quasi-random property, regular tournaments.

The author is supported by Grant-in-Aid for JSPS Fellows 18 J11282 of the Japan Society for the Promotion of Science. 
provide some examples of regular tournaments satisfying the quasi-random property which are also solutions to the problem by Erdös, Moon and Spencer. At last, in Section 5, we discuss another random-like property defined as an adjacency property.

\section{The QUASI-RANDOM PROPERTY AND RELATED FACTS}

In this section, we review the quasi-random property and some related known facts. For a digraph $D$, let $V(D)$ and $E(D)$ be the vertex and the edge set of $D$, respectively. For two distinct vertices $x$ and $y$, let the ordered pair $(x, y)$ denote the edge directed from $x$ to $y$.

First, we give the definition of the quasi-random property of tournaments which was formulated by Chung-Graham [8].

Definition 2.1 (The quasi-random property, [8]). Let $T$ be a tournament with $n$ vertices. Let $\sigma$ be a bijection from $V(T)$ to $\{1,2, \ldots, n\}$. An edge $(x, y)$ of $T$ is called consistent with $\sigma$ if $\sigma(x)<\sigma(y)$. Let $C(T, \sigma)$ be the number of consistent edges with $\sigma$ and $C(T)=\max _{\sigma} C(T, \sigma)$. Then, $T$ has the quasi-random property if $T$ satisfies

$$
C(T) \leq(1+o(1)) \frac{n^{2}}{4} .
$$

Surprisingly, Chung-Graham 8] gave some other properties which are seemingly unrelated, but actually equivalent with (2.1). The interested reader is referred to 8 .

Consistent edges of tournaments was originally investigated by ErdősMoon [14]. Their work was from paired comparisons (e.g. [18]). It is reasonable to find suitable rankings, that is, bijections with many consistent edges. First observe that for every tournament $T$ with $n$ vertices,

$$
\frac{1}{2}\left(\begin{array}{l}
n \\
2
\end{array}\right) \leq C(T) \leq\left(\begin{array}{l}
n \\
2
\end{array}\right) \text {. }
$$

The lower bound of $C(T)$ is obtained by the following simple fact:

$$
C(T, \sigma)+C\left(T, \sigma^{\prime}\right)=\left(\begin{array}{l}
n \\
2
\end{array}\right),
$$

where $\sigma^{\prime}$ is the reversed ranking of $\sigma$ which is defined as $\sigma^{\prime}(v)=n+1-\sigma(v)$ for each $v \in V(T)$. For the upper bound of $C(T)$, the equality holds if and only if $T$ is a transitive tournament. On the other hand, it is nontrivial to check the tightness of the lower bound of $C(T)$. In [14], it was proved that there exist tournaments $T$ such that $C(T) \leq(1+o(1))\left(\begin{array}{l}n \\ 2\end{array}\right) / 2$ by a probabilistic argument. Moreover Spencer [29, 30, and de la Vega [1] proved that random tournament $\mathcal{T}_{n}$ a.a.s satisfies the following property which is stronger than the quasi-random property:

$$
C\left(\mathcal{T}_{n}\right) \leq \frac{1}{2}\left(\begin{array}{l}
n \\
2
\end{array}\right)+O\left(n^{\frac{3}{2}}\right)
$$


Erdős-Moon [14] and Spencer [31] mentioned the problem on explicit constructions of tournaments $T$ such that $C(T)$ is close to the lower bound. At present, such a construction of tournaments $T$ giving the best known "constructive" upper bound of $C(T)$ is obtained by Alon-Spencer [1]. For a prime $p \equiv 3(\bmod 4)$, the Paley tournament $T_{p}$ is the tournament with vertex set $\mathbb{F}_{p}$, the finite field of $p$ elements, and edge set formed by all edges $(x, y)$ such that $x-y$ is a non-zero square of $\mathbb{F}_{p}$. In [1, Theorem 9.1.1], it was proved that

$$
C\left(T_{p}\right) \leq \frac{1}{2}\left(\begin{array}{l}
p \\
2
\end{array}\right)+O\left(p^{\frac{3}{2}} \log p\right) .
$$

In Section 4, by applying the main theorem proved in the next section, we give some new explicit constructions of regular tournaments $T$ with $n$ vertices such that $C(T)$ is close to the lower bound.

\section{MAIN THEOREM}

In this section, we prove our main theorem. We first give the definition of regular digraphs and the adjacency matrix of a digraph. A digraph is said to be $d$-regular if in-degree and out-degree of each vertex is $d$. Especially a tournament with $n$ vertices is simply said to be regular if it is $(n-1) / 2$ regular. The adjacency matrix $M_{D}$ of a digraph $D$ with vertices is the $\{0,1\}$-square matrix of size $n$ whose rows and columns are indexed by the vertices of $D$ and the $(x, y)$-entry is equal to 1 if and only if $(x, y) \in E(D)$.

The following is our main theorem.

Theorem 3.1. Let $T$ be a regular tournament with $n$ vertices. Suppose that the adjacency matrix $M_{T}$ of $T$ has eigenvalues such that $(n-1) / 2=$ $\lambda_{1}, \lambda_{2}, \cdots, \lambda_{n}$. Let $\lambda(T)=\max _{2 \leq i \leq n}\left|\lambda_{i}\right|$. Then,

$$
C(T) \leq \frac{1}{2}\left(\begin{array}{l}
n \\
2
\end{array}\right)+\lambda(T) \cdot n \log _{2}(2 n) .
$$

Remark 3.2. Theorem 3.1 implies that every regular tournament $T$ with $n$ vertices such that $\lambda(T)=o(n / \log n)$ has the quasi-random property. It should be remarked that Kalyanasundaram-Shapira [19] shows a stronger result; a proof of Lemma 2.3 and the first concluding remark in [19] implies that a regular tournament $T$ with $n$ vertices has the quasi-random property if and only if $T$ satisfies that $\lambda(T)=o(n)$. (In [19], the authors considered the eigenvalues of the $\{0, \pm 1\}$-matrix $2 M_{T}-J_{n}+I_{n}$, but these eigenvalues can be directly computed from ones of $M_{T}$.)

On the other hand, Theorem 3.1 not only gives a spectral condition for the quasi-random property, but also implies that estimating eigenvalues of $M_{T}$ provides better upper bounds of $C(T)$ than the bound (2.1). Thus, considering (2.4), Theorem 3.1 provides a spectral condition for a property, which random tournaments a.a.s. satisfy, stronger than the quasi-random property; for example, if $T$ satisfies $\lambda(T)=o(n / \log n)$, then Theorem 3.1 
implies that $C(T) \leq\left(\begin{array}{c}n \\ 2\end{array}\right) / 2+o\left(n^{2}\right)$, which immediately implies the quasirandom property.

In the proof of Theorem 3.1, we use the expander-mixing lemma for normal regular digraphs proved by $\mathrm{Vu}$ [33. A digraph $D$ is said to be normal if $M_{D}$ and its transpose $M_{D}^{t}$ are commutative. In other word, $D$ is normal if $\left|N^{+}(x, y)\right|=\left|N^{-}(x, y)\right|$ for any two distinct vertices $x$ and $y$ where $N^{+}(x, y)$ (resp. $\left.N^{-}(x, y)\right)$ is the set of vertices $z$ such that $(x, z),(y, z) \in E(D)$ (resp. $(z, x),(z, y) \in E(D))$.

Now we are ready to introduce the expander-mixing lemma for normal regular digraphs.

Lemma 3.3 (Expander-mixing lemma, 33]). Let $D$ be a normal d-regular digraph with $n$ vertices and $\lambda(D)=\max _{2 \leq i \leq n}\left|\lambda_{i}\right|$. For two disjoint subsets $A, B \subset V(D)$, let

$$
e(A, B):=|\{(a, b) \in E(D) \mid a \in A, b \in B\}| .
$$

Then for every pair of two disjoint subsets $A, B \subset V(D)$, it holds that

$$
\left|e(A, B)-\frac{d}{n} \cdot\right| A|\cdot| B|| \leq \lambda(D) \sqrt{|A| \cdot|B|} .
$$

From this lemma, we can easily obtain the following corollary.

Corollary 3.4. Let $D$ be a normal d-regular digraph with $n$ vertices. Then for every pair of two disjoint subsets $A, B \subset V(D)$,

$$
|e(A, B)-e(B, A)| \leq 2 \lambda(D) \sqrt{|A| \cdot|B|} .
$$

Proof. From the triangle inequality, we see that

$$
\begin{aligned}
|e(A, B)-e(B, A)| & =\left|\left(e(A, B)-\frac{d}{n} \cdot|A| \cdot|B|\right)-\left(e(B, A)-\frac{d}{n} \cdot|B| \cdot|A|\right)\right| \\
& \leq\left|e(A, B)-\frac{d}{n} \cdot\right| A|\cdot| B||+\left|e(B, A)-\frac{d}{n} \cdot\right| B|\cdot| A|| .
\end{aligned}
$$

Thus, by Lemma 3.3, we get the corollary.

By Corollary 3.4, we get the following lemma.

Lemma 3.5. Let $T$ be a regular tournament with $n$ vertices and let $\sigma$ be a bijection from $V(T)$ to $\{1,2, \ldots, n\}$. Then

$$
C(T, \sigma)-C\left(T, \sigma^{\prime}\right) \leq 2 \lambda(T) \cdot n \log _{2}(2 n) .
$$

Proof of Lemma 3.5. The lemma follows by combining Corollary 3.4 and the argument in [1, pp.150-151] to prove the bound (2.5) for Paley tournaments. It should be noted (see also 6]) that every regular tournament $T$ with $n$ vertices is normal since it holds that $M_{T}^{t}=J_{n}-I_{n}-M_{T}$, where $I_{n}$ and $J_{n}$ are the identity matrix and the all-one matrix of order $n$, respectively.

Fix a bijection $\sigma$. Let $r$ be the smallest integer such that $n \leq 2^{r}$. Let $n=a_{1}+a_{2}$, where $a_{1}$ and $a_{2}$ are positive integers with $a_{1}, a_{2} \leq 2^{r-1}$. Consider a partition of $V(T)$, say $A_{1}$ and $A_{2}$, such that $A_{1}$ is the set of 
"highly ranked" $a_{1}$ vertices in $\sigma$ and $A_{2}$ is the remaining $a_{2}$ vertices. It follows from Corollary 3.4 that

$$
e\left(A_{1}, A_{2}\right)-e\left(A_{2}, A_{1}\right) \leq 2 \lambda(T) \sqrt{a_{1} a_{2}} \leq 2 \lambda(T) \cdot 2^{r-1} .
$$

Next, let $a_{1}=a_{11}+a_{12}$, where $a_{11}$ and $a_{12}$ are positive integers with $a_{11}, a_{12} \leq 2^{r-2}$, and similarly for $a_{2}=a_{21}+a_{22}$. As above, divide $A_{1}$ into two subsets, say $A_{11}$ and $A_{12}$, where $A_{11}$ is the set of "highly ranked" $a_{11}$ vertices of $A_{1}$ in $\sigma$ and $A_{12}$ is the remaining $a_{12}$ vertices of $A_{1}$. For $a_{21}$ and $a_{22}$, two subsets $A_{21}$ and $A_{22}$ of $A_{2}$ are defined in the same way as $A_{11}, A_{12}$. It then follows from Corollary 3.4 that

$$
\begin{aligned}
& e\left(A_{11}, A_{12}\right)-e\left(A_{12}, A_{11}\right)+e\left(A_{21}, A_{22}\right)-e\left(A_{22}, A_{21}\right) \\
& \leq 2 \lambda(T) \sqrt{a_{11} a_{12}}+2 \lambda(T) \sqrt{a_{21} a_{22}} \\
& \leq 2 \cdot 2 \lambda(T) \cdot 2^{r-2} .
\end{aligned}
$$

Then iterate such estimation from the first to the $r$-th step. In the $i$-th step, $V(T)$ is partitioned into $2^{i}$ subsets, say $A_{\varepsilon 1}$ and $A_{\varepsilon 2}\left(\varepsilon \in\{1,2\}^{i}\right)$, such that each $A_{\varepsilon j}(j=1,2)$ contains at most $2^{r-i}$ vertices which are consecutive in $\sigma$. It follows from Corollary 3.4 that

$$
\sum_{\varepsilon \in\{1,2\}^{i-1}}\left\{e\left(A_{\varepsilon 1}, A_{\varepsilon 2}\right)-e\left(A_{\varepsilon 2}, A_{\varepsilon 1}\right)\right\} \leq 2^{i-1} \cdot 2 \lambda(T) \cdot 2^{r-i}=2 \lambda(T) \cdot 2^{r-1} .
$$

On the other hand, it turns out from the construction of partitions that

$$
\sum_{1 \leq i \leq r} \sum_{\varepsilon \in\{1,2\}^{i-1}}\left\{e\left(A_{\varepsilon 1}, A_{\varepsilon 2}\right)-e\left(A_{\varepsilon 2}, A_{\varepsilon 1}\right)\right\}=C(T, \sigma)-C\left(T, \sigma^{\prime}\right) .
$$

Thus by combining (3.6) and (3.7), it follows that

$$
C(T, \sigma)-C\left(T, \sigma^{\prime}\right) \leq r \cdot 2 \lambda(T) \cdot 2^{r-1} \leq 2 \lambda(T) \cdot n \log _{2}(2 n) .
$$

Proof of Theorem [3.1. The theorem is a direct consequence of the equality (2.3) and Lemma 3.5 .

Remark 3.6. It should be noted that for every regular tournament $T$ with $n$ vertices, $\lambda(T) \cdot n \log _{2}(2 n)$ cannot be less than $\sqrt{n^{3}+n} \log _{2}(2 n) / 2$. In fact, for every such tournament $T$, it holds that

$$
\lambda(T) \geq \frac{\sqrt{n+1}}{2} .
$$

Indeed, for every strongly-connected normal $d$-regular digraph $D$ with $n$ vertices, it holds that

$$
n d=E(D)=\operatorname{Tr}\left(M_{D} M_{D}^{t}\right)=\sum_{i=1}^{n}\left|\lambda_{i}\right|^{2} \leq d^{2}+(n-1) \lambda(D)^{2},
$$

which follows from the hand shaking lemma and the Perron-Frobenius theorem (see e.g. [21]). The idea of the above inequality can be found in [20, 
p.217]. Also note that every regular tournament $T$ is strongly connected, which follows from the Perron-Frobenius theorem and facts that $T$ is normal and every eigenvalue of $M_{T}$ corresponding to eigenvectors distinct to the all-one vector has the real part equal to $-1 / 2$ (see also [5]).

\section{EXAMPLES OF QUASI-RANDOM REGULAR TOURNAMENTS}

In this section, we give some examples of regular tournaments $T$ with $n$ vertices and $\lambda(T)=o(n / \log n)$. As will be shown below, we can construct such tournaments for almost all positive integers $n$.

First we consider the following tournaments constructed from finite fields which are variants of cyclotomic tournaments (see e.g. 24] and reference therein). Let $m$ be a positive even integer and $p \equiv m+1(\bmod 2 m)$ be a prime. Note that there exist infinitely many such primes by the Dirichlet's theorem on arithmetic progressions and the fact that $m+1$ and $2 m$ are coprime when $m$ is even. Recall that $\mathbb{F}_{p}$ is the finite field of order $p$. Let $g$ be a primitive element of $\mathbb{F}_{p}$. For even $m$, the multiplicative group of $\mathbb{F}_{p}$, which is denoted by $\mathbb{F}_{p}^{*}$, is divided into $m$ cosets $S_{0}, S_{1}, \ldots, S_{m-1}$ where $S_{i}:=\left\{g^{t} \mid t \equiv i(\bmod m)\right\}$ for each $0 \leq i \leq m-1$. Note that $S_{j}=-S_{i}$ if $j \equiv-i(\bmod m)$.

Definition 4.1. Let $\boldsymbol{i}=\left(i_{1}, i_{2}, \ldots, i_{m / 2}\right) \in\{0,1, \ldots, m-1\}^{m / 2}$ such that $S_{\boldsymbol{i}}=S_{i_{1}} \cup \cdots \cup S_{i_{m / 2}}$ and $\mathbb{F}_{p}^{*} \backslash S=-S$. Then the tournament $T_{p}^{m}\left(S_{\boldsymbol{i}}\right)$ is defined as follows:

$$
\begin{aligned}
& V\left(T_{p}^{m}\left(S_{\boldsymbol{i}}\right)\right)=\mathbb{F}_{p}, \\
& E\left(T_{p}^{m}\left(S_{\boldsymbol{i}}\right)\right)=\left\{(x, y) \in \mathbb{F}_{p}^{2} \mid x-y \in S_{\boldsymbol{i}}\right\} .
\end{aligned}
$$

This is a direct generalization of Paley tournament since $T_{p}^{m}\left(S_{\boldsymbol{i}}\right)$ is exactly $T_{p}$ in the case of $m=2$. Moreover from the definition, it is not so hard to see that $T_{p}^{m}\left(S_{\boldsymbol{i}}\right)$ is a regular tournament with $p$ vertices.

Now we obtain the following corollary.

\section{Corollary 4.2.}

$$
C\left(T_{p}^{m}\left(S_{\boldsymbol{i}}\right)\right) \leq \frac{1}{2}\left(\begin{array}{l}
p \\
2
\end{array}\right)+O\left(p^{\frac{3}{2}} \log p\right) .
$$

Corollary 4.2 is proved by combining Lemma 3.5 and the following evaluation of $\lambda\left(T_{p}^{m}\left(S_{\boldsymbol{i}}\right)\right)$.

\section{Lemma 4.3.}

$$
\lambda\left(T_{p}^{m}\left(S_{i}\right)\right) \leq \frac{m \sqrt{p}}{2} .
$$

Proof. First, by a simple calculation, it can be shown that the set of eigenvalue of $M_{T_{p}^{m}\left(S_{\boldsymbol{i}}\right)}$ is

$$
\left\{\sum_{s \in S_{\boldsymbol{i}}} \psi(s) \mid \psi \text { is an additive character of } \mathbb{F}_{p}\right\} .
$$


Since $S_{i}=g^{i} S_{0}$ for each $1 \leq i \leq m-1$, we see that

$$
\sum_{s \in S_{i}} \psi(s)=\sum_{s \in g^{i} S_{0}} \psi(s)=\sum_{s \in S_{0}} \psi\left(g^{i} s\right) .
$$

Since $S_{0}$ is the set of non-zero $m$-th power elements and each non-zero $m$-th power residue appears exactly $m$ times in the sequence $\left(x^{m}\right)_{x \in \mathbb{F}_{p}^{*}}$,

$$
\sum_{s \in S_{0}} \psi\left(g^{i} s\right)=\frac{1}{m} \sum_{x \in \mathbb{F}_{p}^{*}} \psi\left(g^{i} x^{m}\right) .
$$

At last, we use the following known estimation (see e.g. [26, p.44]);

$$
\left|\sum_{x \in \mathbb{F}_{p}} \psi\left(a x^{m}\right)\right| \leq(m-1) \sqrt{p}
$$

for any non-trivial additive character $\psi$ and $a \neq 0$. By combining (4.4), (4.5) and (4.6),

$$
\lambda\left(T_{p}^{m}\left(S_{i}\right)\right) \leq \frac{m}{2} \cdot \frac{1}{m} \cdot\{(m-1) \sqrt{p}+1\}=\frac{(m-1) \sqrt{p}+1}{2} \leq \frac{m \sqrt{p}}{2} .
$$

The second example is doubly regular tournament which has been extensively studied in algebraic combinatorics and related areas (e.g. [23]).

Definition 4.4. A tournament $T$ with $n$ vertices is called a doubly regular tournament if $T$ is a regular tournament such that for any distinct two vertices $x$ and $y, N^{+}(x, y)=N^{-}(x, y)=(n-3) / 4$.

Let $D R T_{n}$ denote a doubly regular tournament with $n$ vertices.

\section{Corollary 4.5.}

$$
C\left(D R T_{n}\right) \leq \frac{1}{2}\left(\begin{array}{l}
n \\
2
\end{array}\right)+O\left(n^{\frac{3}{2}} \log n\right) .
$$

Corollary 4.5 is proved by the following well-known evaluation of $\lambda\left(D R T_{n}\right)$ which also shows that the inequality (3.8) is tight.

Lemma 4.6 (e.g. [10]).

$$
\lambda\left(D R T_{n}\right)=\frac{\sqrt{n+1}}{2} .
$$

Proof. We give a proof for the reader's convenience. Let $M=M_{D R T_{n}}$. Then by the definition, it holds that

$$
M M^{t}=\frac{n+1}{4} I_{n}+\frac{n-3}{4} J_{n} .
$$

Since $M+M^{t}=J_{n}-I_{n}$, we obtain the following equality.

$$
M^{2}+M+\frac{n+1}{4} I_{n}-\frac{n+1}{4} J_{n}=O .
$$


Since $D R T_{n}$ is regular, we see that $(n-1) / 2$ is an eigenvalue of $M$ and a corresponding eigenvector is the all-one eigenvector 1 . Since $D R T_{n}$ is normal, each eigenvalue $\theta$ except for $(n-1) / 2$ has an eigenvector $\boldsymbol{v}$ which is orthogonal to $\mathbf{1}$. Thus,

$$
\left(\theta^{2}+\theta+\frac{n+1}{4}\right) \boldsymbol{v}=\mathbf{0} .
$$

Since $\boldsymbol{v} \neq \mathbf{0}$, we get

$$
\left(\theta^{2}+\theta+\frac{n+1}{4}\right)=0
$$

completing the proof.

Remark 4.7. We remark that Corollary 4.5 is a generalization of the bound (2.5) because Paley tournaments are also doubly-regular tournaments. For other non-isomorphic examples of doubly regular tournaments, see e.g. [17] and [32. As shown in, for example, [16] and [23], there are some known constructions of doubly regular tournaments such that the number of vertices is non-prime (and non-prime power). Especially, constructions of complex codebooks in [16] provide $D R T_{n}$ for every integer $n$ such that each prime factor $f$ of $n$ is the form of $f \equiv 3(\bmod 4)$.

Remark 4.8. By the definition of $D R T_{n}, n$ must be a positive integer of the form $n \equiv 3(\bmod 4)$. On the other hand, as an analogue of $D R T_{n}$ for integers $n$ of the form $n \equiv 1(\bmod 4)$, Savchenko [24] introduced the notion of a nearly-doubly-regular tournament $C N D R_{n}$ with $n$ vertices which is a certain regular tournament with exactly four eigenvalues distinct to $(n-1) / 2$ with multiplicity $(n-1) / 4$. According to [24], it holds that $\lambda\left(C N D R_{n}\right)=$ $(\sqrt{n}+1) / 2$. Thus if there exists $C N D R_{n}$ for infinitely many $n \equiv 1(\bmod 4)$, then it holds that

$$
C\left(C N D R_{n}\right) \leq \frac{1}{2}\left(\begin{array}{l}
n \\
2
\end{array}\right)+O\left(n^{\frac{3}{2}} \log n\right) .
$$

It is conjectured in 24] (see also [25]) that there exists a $C N D R_{n}$ for every $n \equiv 1(\bmod 4)$. Interestingly, Savchenko [24] also found examples of $C N D R_{p}$ for primes $p=5,13,29,53,173,229,293$ and 733 from the class of $T_{p}^{4}\left(S_{(0,1)}\right)$ in the first example, and thus Lemma 4.3 can be improved for these examples. (It is shown in 24 that for every prime $p \equiv 5(\bmod 8)$, $T_{p}^{4}\left(S_{(0,1)}\right)$ has exactly four eigenvalues distinct to $(p-1) / 2$ with multiplicity $(p-1) / 4$.) It would be interesting to prove or disprove the existence of infinitely many primes $p \equiv 5(\bmod 8)$ such that the tournament $T_{p}^{4}\left(S_{(0,1)}\right)$ is in the class of $C N D R_{p}$.

The third example is based on a construction of pseudo-random graphs due to Shparlinski [27]. For related facts on eliptic curves, see [27, Section 2.1]. For a prime $p$, let $n \in[p+1-2 \sqrt{p}, p+1+2 \sqrt{p}]$ be an odd integer. It is known (e.g. [7, [12]) that there exists an eliptic curve $E$ over $\mathbb{F}_{p}$ such that the number of $\mathbb{F}_{p}$-rational points of $E$ is $n$. It is also known (e.g. [28]) 
that all $\mathbb{F}_{p}$-rational points of $E$ form an abelian group $G$ of order $n$ under an operation $\oplus$. Let $0_{G}$ be the identity of $G$. For an element $s \in G$ and a subset $S \subset G$, the inverse of $s$ is denoted by $\ominus s$ and let $\ominus S=\{\ominus s \mid s \in S\}$.

Definition 4.9. Let $S \subset G$ be a subset such that $S \cup \ominus S \cup\left\{0_{G}\right\}=G$ and $|S|=(n-1) / 2$. Then the tournament $T_{p, n}(S)$ is defined as follows.

$$
\begin{aligned}
& V\left(T_{p, n}(S)\right)=G, \\
& E\left(T_{p, n}(S)\right)=\left\{(x, y) \in G^{2} \mid x \ominus y \in S\right\} .
\end{aligned}
$$

By the definition, $T_{p, n}(S)$ is a regular tournament with $n$ vertices.

Corollary 4.10. There exists a subset $S \subset G$ such that

$$
C\left(T_{p, n}(S)\right) \leq \frac{1}{2}\left(\begin{array}{l}
n \\
2
\end{array}\right)+O\left(n^{\frac{3}{2}} \log ^{2} n\right) .
$$

Corollary 4.10 is obtained by Lemma 3.5 and the following evaluation of $\lambda\left(T_{p, n}(S)\right)$ which follows from [27, Theorem 1].

Lemma 4.11 ([27]). There exists a subset $S \subset G$ such that

$$
\lambda\left(T_{p, n}(S)\right)=O(\sqrt{n} \log n) .
$$

For the details of a construction of such a subset $S$, see [27].

Remark 4.12. It is worth noting that as shown in 27, almost all positive integers are in the interval $[p+1-2 \sqrt{p}, p+1+2 \sqrt{p}]$ for some prime $p$. Indeed, it holds $([27])$ that

$\lim _{N \rightarrow \infty} \frac{\mid\{n \leq N \mid \exists \text { prime } p \text { s.t. } n \text { is odd and } n \in[p+1-2 \sqrt{p}, p+1+2 \sqrt{p}]\} \mid}{\left\lceil\frac{N}{2}\right\rceil}=1$.

Thus the third example provides regular tournaments $T$ with $n$ vertices and small $\lambda(T)$ for almost all positive integers $n$.

\section{ShÜtTE'S PROBLEM FOR TOURNAMENTS}

At last, in this section, we focus on another random-like property.

Definition 5.1. Let $k$ be a positive integer. A tournament $T$ has the property $S_{k}$ if for every $A \subset V(T)$ of size $k$, there exists a vertex $z \notin A$ directing to all members of $A$.

The Shütte's problem asks the existence of tournaments satisfying this property (see [13] and [22]). As shown by Erdős [13], random tournaments a.a.s. satisfy $S_{k}$ for any $k \geq 1$. On the other hand, the problem of explicit constructions has been considered in graph theory. For example, Graham-Spencer [15] showed that the Paley tournament $T_{p}$ satisfies $S_{k}$ if $p>k^{2} 2^{2 k-2}$ for each $k \geq 1$. From the digraphs constructed in [3], we can also construct tournaments satisfying $S_{k}$ for every $k$ by adding some edges. At present, there seems to be almost no explicit constructions of tournaments satisfying both of the quasi-random property and $S_{k}$ except for Paley 
tournaments. The following proposition and Corollary 4.2 show that the tournament $T_{p}^{m}\left(S_{\boldsymbol{i}}\right)$ has the quasi-random property and $S_{k}$.

Proposition 5.2. Let $m$ be an even positive integer. Then for every $k \geq$ 1 , there exists a prime $p_{m}(k)$ such that for every prime $p>p_{m}(k)$, the tournament $T_{p}^{m}\left(S_{i}\right)$ has the property $S_{k}$.

Proposition 5.2 is proved by a direct generalization of the discussion in [15] and 2], so we omit the proof here. Moreover, it is not so hard to prove that $T_{p}^{m}\left(S_{i}\right)$ has the existentially closed property (see e.g. [4]).

We also note that doubly regular tournaments constructed in [32] satisfy both of the quasi-random property and $S_{2}$, which follows from Corollary 4.5 and the corollary in [32, p.277].

\section{ACKNOWLEDGEMENT}

We would like to thank Masanori Sawa and Yujie Gu for their valuable comments. We also greatly appreciate Sergey Savchenko for his helpful remarks.

\section{REFERENCES}

[1] N. Alon, J. H. Spencer, The Probabilistic Method, Fourth edition, John Wiley \& Sons, Inc., Hoboken, NJ, 2016.

[2] W. Ananchuen, L. Caccetta, Cubic and quadruple Paley graphs with the $n$-e.c. property, Discrete Math. 306 (2006), 2954-2961.

[3] Y. Anbalagan, H. Huang, S. Lovett, S. Norin, A. Vetta, H. Wu, Large supports are required for well-supported Nash equilibria, in Proceedings of the 18th International Workshop on Approximation Algorithms for Combinatorial Optimization Problems, APPROX15, LIPIcs. Leibniz Int. Proc. Inform., 40, N. Garg, K. Jansen, A. Rao and J. D. P. Rolim, eds., Wadern, 2015, pp. 78-84.

[4] A. Bonato, The search for N-e.c. graphs, Contrib. Discrete Math. 4 (2009), 40-53.

[5] A. Brauer, I. C. Gentry, On the characteristic roots of tournament matrices, Bull. Amer. Math. Soc. 74 (1968), 1133-1135.

[6] A. Brauer, I. C. Gentry, Some remarks on tournament matrices, Linear Algebra Appl. 5 (1972), 311-318.

[7] R. Bröker, P. Stevenhagen, Elliptic curves with a given number of points. Lecture Notes in Comput. Sci. 3076 (2014), 117-131.

[8] F. R. K. Chung, R. L. Graham, Quasi-random tournaments, J. Graph Theory 15 (1991), 173-198.

[9] L. N. Coregliano, A. A. Razborov, On the density of transitive tournaments, J. Graph Theory 85 (2017), 12-21.

[10] D. de Caen, D. A. Gregory, S. J. Kirkland, N. J. Pullman, J. S. Maybee, Algebraic multiplicity of the eigenvalues of a tournament matrix, Linear Algebra Appl. 169 (1992), 179-193.

[11] W. F. de la Vega, On the maximum cardinality of a consistent set of arcs in a random tournament, J. Combin. Theory Ser. B 35 (1983), 328-332.

[12] M. Deuring, Die Typen der Multiplikatorenringe elliptischer Funktionenkörper. Abh. Math. Sem. Hansischen Univ. 14 (1941), 197-272.

[13] P. Erdős, On a problem in graph theory, Math. Gaz. 47 (1963), 220-223.

[14] P. Erdős, J. W. Moon, On sets of consistent arcs in a tournament, Canad. Math. Bull. 8 (1965), 269-271. 
[15] R. L. Graham, J. H. Spencer, A constructive solution to a tournament problem, Canad. Math. Bull. 14 (1971), 45-48.

[16] H. Hu, J. Wu, New constructions of codebooks nearly meeting the Welch bound with equality. IEEE Trans. Inf. Theory 60 (2014), 1348-1355.

[17] N. Ito, T. Okamoto, Note on Hadamard tournaments of Szekeres type, Graphs Combin. 10 (1994), 47-51.

[18] M. G. Kendall, B. B. Smith, On the method of paired comparisons, Biometrika 31 (1940), 324-345.

[19] S. Kalyanasundaram, A. Shapira, A note on even cycles and quasirandom tournaments, J. Graph Theory 73 (2013), 260-266.

[20] M. Krivelevich, B. Sudakov, Pseudo-random graphs, in More sets, graphs and numbers, Bolyai Soc. Math. Stud., 15, Springer-Verlag, Berlin, 2006, pp. 199-262.

[21] J. H. van Lint, R. M. Wilson, A Course in Combinatorics, Second edition, Cambridge University Press, Cambridge, 2001.

[22] J. W. Moon, Topics on Tournaments, Holt, Rinehart and Winston, New YorkMontreal, 1968.

[23] K. B. Reid, E. Brown, Doubly regular tournaments are equivalent to skew Hadamard matrices, J. Combinatorial Theory Ser. A 12 (1972), 332-338.

[24] S. V. Savchenko, On 5-cycles and 6-cycles in regular $n$-tournaments, J. Graph Theory 83 (2016), 44-77.

[25] S. V. Savchenko, On the number of 7-cycles in regular $n$-tournaments, Discrete Math. 340 (2017), 264-285.

[26] W. M. Schmidt, Equations over Finite Fields. An Elementary Approach, Lecture Notes in Mathematics, Vol. 536, Springer-Verlag, Berlin-New York, 1976.

[27] I. E. Shparlinski, Pseudorandom graphs with elliptic curves. Lecture Notes in Comput. Sci. 4957 (2008), 284-292.

[28] J. H. Silverman, The Arithmetic of Elliptic Curves Springer, Berlin, 1995.

[29] J. Spencer, Optimal ranking of tournaments, Networks 1 (1971), 135-138.

[30] J. Spencer, Optimally ranking unrankable tournaments, Period. Math. Hungar. 11 (1980), 131-144.

[31] J. Spencer, Probabilistic methods, Graphs Combin. 1 (1985), 357-382.

[32] G. Szekeres, Tournaments and Hadamard matrices, Enseignement Math. (2) 15 (1969), 269-278.

[33] V. H. Vu, Sum-product estimates via directed expanders, Math. Res. Lett. 15 (2008), $375-388$.

Graduate School of System Informatics, Kobe University, Rokkodai 1-1, NADA, KoBE, 657-8501, JAPAN

E-mail address: 155x601x@stu.kobe-u.ac.jp 